\title{
Posterior fossa decompression and the cerebellum in Chiari type II malformation: a preliminary MRI study
}

\author{
Michael S. Salman \\ Section of Pediatric Neurology, Children's Hospital, AE 308, 820 Sherbrook Street, Winnipeg, MB \\ R3A 1R9, Canada. Faculty of Medicine, University of Manitoba, Winnipeg, MB, Canada
}

Michael S. Salman: msalman@hsc.mb.ca

\begin{abstract}
Objectives-Chiari type II malformation (CII) is a congenital deformity of the hindbrain. The posterior fossa and cerebellum are small in CII. The cerebellar atrophy is associated with cognitive and motor deficits. Brainstem compression occurs in some patients with CII for whom posterior fossa decompression may be life saving. The aim was to determine whether posterior fossa decompression can prevent or reduce the cerebellar atrophy in CII.
\end{abstract}

Methods-Cerebellar volumes and their tissue types (gray matter, white matter, and CSF volumes) from brain MRI were compared among four CII patients, aged 9.5 to 16.5 years, who had had posterior fossa decompression in infancy, 28 CII patients who had not had posterior fossa decompression, and ten age-matched normal controls. Parametric and non-parametric tests investigated group differences.

Results-Compared to controls, mean cerebellar volume was significantly smaller in CII patients $(p<0.0001)$. Mean CSF volume within the cerebellar fissures and fourth ventricle was significantly smaller in patients without posterior fossa decompression compared to the CII patients who had the decompression, $p=0.043$. Mean CSF volume of the latter group was similar to the controls. Other cerebellar volumetric measurements did not differ between the CII groups.

Conclusions-Posterior fossa decompression normalizes CSF spaces within the posterior fossa in CII but does not prevent the cerebellar atrophy. The author proposes that surgical expansion of the posterior fossa should be considered in infants with CII who have a significantly small posterior fossa, to prevent or reduce the deficits associated with the cerebellar atrophy.

\section{Keywords}

Chiari II malformation; Cerebellum; MRI; Atrophy

\section{Introduction}

Chiari type II malformation (CII) is an almost universal feature of myelomeningocele (MMC), the most common and severe type of spina bifida [1]. The hindbrain is dysplastic in CII [2,3] because the posterior fossa is small and its contents are distorted [4]. The cerebellar vermis herniates upward through the tentorial incisura and downward through foramen magnum. The cerebellum is reduced in size [4]. The lower brainstem is displaced

Correspondence to: Michael S. Salman, msalman@hsc.mb.ca.

Conflict of interest The author declares no conflict of interest. 
downwards [2]. Supratentorial abnormalities, including primary dysgenesis of the corpus callosum, occur commonly in CII [4].

This paper explores two questions about the structural changes associated with CII in patients with MMC. The first question is whether, on the basis of MRI information, posterior fossa decompression can prevent or reduce the cerebellar atrophy in CII. The paper considers data on MRI-derived cerebellar volumes in patients with CII who had posterior fossa decompression during the first 2 years of life, patients with CII who had not had posterior fossa decompression, and age-matched controls. The second question is whether surgical expansion of the posterior fossa should be performed in early infancy in selected patients with MMC to reduce or ameliorate the cerebellar atrophy. This is presented in the form of a proposal and a model of the mechanisms of cerebellar atrophy in CII.

\section{Structural changes in Chiari type II malformation}

Several studies have documented a reduction in cerebellar size in CII in utero [5,6], infancy, and early childhood [7,8]. There is a reduction in: (1) the number of cells in the vermis [9], (2) cerebellar weight [10], (3) cerebellar volume [11], and (4) cerebellar and cerebrum volumes in children with upper compared to lower spinal lesions [12].

A recent study in CII using cerebellar parcellation techniques on MRI quantified the size of the cerebellar lobes [13]. Individuals with CII had an overall reduction in cerebellar size; in addition, compared to controls, they showed a smaller absolute volume and volume ratio of the gray matter of the inferior posterior cerebellar lobes and a larger absolute volume and volume ratio of the gray matter of the anterior lobes $[13,14]$. The significance of enlarged anterior lobes is uncertain.

The overall small cerebellar size in CII has been attributed to atrophy from crowding and mechanical compression caused by a small posterior fossa size. Hindbrain herniation obstructs CSF outflow from the fourth ventricle and commonly causes hydrocephalus in MMC [15]. Hydrocephalus may exacerbate hindbrain compression within the posterior fossa.

Gross pathology and biometric analyses have identified significant variations in hindbrain shape in CII $[3,16]$. Linear measurements and planimetry (area measurements) of the posterior fossa and the vermis have been reported in CII. The mean area of the midsagittal anterior, posterior superior, and inferior vermis lobules was each larger in CII patients in comparison with age-matched controls [17]. No group differences in these areas were detected between CII patients who had posterior fossa decompression and CII patients who did not have the decompression.

The most likely explanation for the midsagittal expansion of the vermis is tissue compression occurring within a small posterior fossa, which generates pressure that squeezes the vermis from either side towards the midline [17,18]. Expansion of the anterior vermis lobules may also be related to the enlarged anterior cerebellar lobes reported recently in CII [13]. The reduction in cerebellar volume in CII affects the cerebellar hemispheres more than the vermis; within the hemispheres, the posterior lobes, in particular, are reduced in volume [13].

Other dysmorphologies occur in CII, some may be related to increased intracranial pressure $[2,4]$. These include atrophy of the corpus callosum and compression of the diencephalon. The brain as a whole may show reduction in the thickness of the cortical mantle, reduced overall brain mass, and selective thinning of the posterior cortical regions [19]. A diffusion 
tensor study of the brain in children with MMC revealed abnormal development, impairment in myelination, and intrinsic axonal abnormalities in the cerebral association pathways [20].

\section{Functional consequences of Chiari type II malformation}

Intrinsic brainstem abnormalities and pressure on the brainstem from a crowded posterior fossa and hydrocephalus cause diverse findings such as headaches, apnea, bradycardia, dysphagia, torticollis, and spasticity [1]. Brainstem compression occurs in a third of infants with CII, and may be fatal in up to a third of these patients [21]. Surgical decompression of the posterior fossa for symptomatic CII infants with brainstem compression is required in $8 \%$ to $17 \%$ of these patients [1], and usually leads to clinical improvement. Fetal surgery for MMC may reduce the incidence and severity of brainstem dysfunction [22].

Speech motor deficits, dysfunction in the speed and accuracy of rhythm perception, cognitive dysfunction, and perceptual and motor timing deficits have been reported in children with MMC [11,12,14,23].

Eye movement abnormalities occur in CII including saccadic smooth pursuit, saccadic dysmetria, vestibulo-ocular reflex abnormalities, nystagmus, strabismus, and internuclear ophthalmoplegia [24]. Eye movement recording revealed that CII patients with abnormal ocular motor functions had a smaller midsagittal posterior fossa areas and their cerebellar volumes were smaller than patients with CII who had normal eye movements [25]; while midsagittal vermis expansion and preserved medial cerebellar volume corresponded to sparing of some eye movement abnormalities [25], despite the overall reduction in cerebellar size in CII.

\section{Is Chiari type II malformation a progressive deformity?}

The hydrocephalus associated with MMC and CII is usually progressive, and is usually shunted soon after birth. However, there is only anecdotal evidence that the deformity of CII per se is progressive. Several factors need to be considered first when disease progression is suspected in children with MMC. For example, shunt malfunction, the development of spinal or bulbar syringomyelia, tethered spinal cord and the presence of an unrelated neurological disorder. The development of symptoms and signs of brainstem compression in some infants with MMC, the appearance of ataxia, weakness, and spasticity in the upper limbs over a period of weeks or months, and degeneration of the cerebellum described on MRI suggest clinical and neuroradiological progression in CII $[1,2,8,21,26]$. There is no direct evidence of progressive hindbrain atrophy documented systematically in a longitudinal MRI study in CII.

The main aim of this preliminary investigation was to determine, on the basis of MRI information, whether posterior fossa decompression can prevent or reduce the cerebellar atrophy in CII.

\section{Methods}

Participants for this investigation were a cohort of patients and normal controls, aged 8-19 years, who were participants in a spina bifida project funded by the National Institute of Child Health and Human Development. The study was approved by the local Research Ethics Board. Cerebellar volumes were measured on brain MRI in four patients with CII who had posterior fossa decompression during the first 2 years of life and compared to agematched ten normal controls and 28 patients with CII who did not have posterior fossa decompression. The MRI scans were performed several years ( $>5$ years) following the decompression surgery. 
MRI images were acquired on 1.5T GE MRI scanner. Details of acquisition, processing, and automatic segmentation of the images have been reported elsewhere [11]. Cerebellar volumes were measured automatically after mask generation. Cerebellar white matter, cerebellar gray matter, and cerebellar CSF (including CSF in the fourth ventricle and cerebellar fissures) volumes, were computed. Medial and lateral cerebellar volumes were also calculated as an approximate measure of vermis and cerebellar hemispheres volumes, respectively [11].

A Statistical Package for Social Sciences, version 15.0 (SPSS Inc., Chicago, Il, USA) was used to analyze the data. Two-tailed, independent Student $t$ tests and Mann-Whitney tests were used to investigate group differences. Significance was defined when $p$ was less than 0.05 .

\section{Results}

Mean total CSF volume within the cerebellar fissures and fourth ventricle was significantly smaller $\left(4,636 \mathrm{~mm}^{3}\right)$ in patients who did not have posterior fossa decompression in comparison with the CII group who had the decompression $\left(6,391 \mathrm{~mm}^{3} p=0.043\right.$, Table 1). The control participants and the patients who had posterior fossa decompression had similar mean cerebellar CSF volumes. In CII patients, other cerebellar volumetric measurements did not differ according to the presence $(N=4)$ or absence $(N=28)$ of posterior fossa decompression.

In comparison to the controls, the CII group had significantly smaller mean total cerebellar volume ( $p<0.0001)$, smaller mean lateral cerebellar volume $(p<0.001)$, and smaller mean gray matter cerebellar volume $(p<0.001)$, either as a whole group or when split according to decompression history. Mean white matter volume was also significantly reduced in the CII group who did not have posterior fossa decompression $(N=28)$ in comparison to the control group $(N=10, p<0.001)$, although the reduction in white matter volume was not significant when the CII group who had posterior fossa decompression $(N=4)$ was compared to the normal control $(N=10, p=0.12$, Table 1$)$.

\section{Discussion}

Posterior fossa decompression appears to be associated with long-term normalization of cerebellar CSF spaces and presumably improved CSF flow within the posterior fossa. However, this surgical procedure offers no obvious long-term benefits for the cerebellum, because cerebellar atrophy was still evident in the CII patients several years after the surgical procedure was performed and was similar to the cerebellar atrophy seen in CII patients who had not had surgical decompression. The CII group had smaller cerebellar volumes, mainly involving the cerebellar hemispheres, than normal controls as reported previously [11]. This investigation also adds to the emerging data showing that CII is associated with both gray and white matter cerebellar atrophy.

Findings from this investigation are consistent with results from the cerebellar planimetry study in CII [17], where an enlarged midsagittal vermis area, which suggests vermis compression, was found in CII patients including those who had posterior fossa decompression. It appears that surgical decompression of the posterior fossa, usually performed in infancy, does not spare the vermis from compression and cerebellar atrophy.

Interestingly, similar findings have been reported in neonates who had fetal MMC repair [27]. Their sub-arachnoid extra-axial supratentorial and posterior fossa CSF spaces were similar to normal controls on postnatal MRI and their fourth ventricle re-appeared, whereas neonates who had the standard postnatal surgical repair of their MMC had significantly 
reduced sub-arachnoid CSF spaces on MRI postnatally. All fetuses with MMC had fetal MRI around midgestation prior to any surgery that showed significantly reduced subarachnoid CSF spaces in comparison to gestation-matched normal fetuses [27]. The same study also reported the persistence of an abnormally reduced brain thickness and cerebellar width on postnatal MRI in all neonates with MMC irrespective of whether the surgery to close their spinal defect was performed in utero or postnatally [27]. Taken together, findings from this investigation and that of Danzer et al. and others [6,27], suggest that hindbrain abnormalities in MMC occur early in utero (and prior to fetal surgical repair) and persist thereafter.

Brainstem compression occurs in severely affected patients. This requires urgent posterior fossa decompression, which appears to open up the CSF spaces within the posterior fossa as suggested by this investigation but the decompression surgery does not expand the size of the posterior fossa significantly [17]. Posterior fossa decompression in CII involves removing or cutting a small piece of skull bone opposite the foramen magnum and not much more above that region in order to avoid the low-lying torcular (confluence of the venous sinuses) in CII (Dr. P. McDonald, personal communication). The posterior fossa size is not "actively" expanded by the surgical decompression, as opposed to a posterior fossa decompression for Chiari type I malformation. It is perhaps not surprising that the procedure did not result in any significant change in the cerebellar MRI quantitative data in this investigation except for showing a larger cerebellar CSF volume. Although the surgical decompression relieves the obstruction to CSF flow through the foramina of Luschka and Magendie caused by vermis herniation, it appears to have little effect on the gross size of the cerebellum.

\section{Should surgical expansion of the posterior fossa be done in early infancy in selected patients with myelomeningocele? A proposal}

The posterior fossa normally undergoes rapid development at 12-weeks gestation [28]; therefore, its permanent smaller size in MMC is determined early and is not reversible by fetal repair of the spinal defect usually performed in midgestation. Figure 1 summarizes findings from this investigation and other studies [17,18,22,27-29] in relationship to normal cerebellar and posterior fossa development (left column). Since the rapid cerebellar growth starts after posterior fossa rapid growth, its development is restricted and adversely affected in CII by the already small size of the posterior fossa (see box with the bold and italics text on the right in Fig. 1). The middle left column of Fig. 1 illustrates MRI findings before and after fetal MMC surgery and relates them to MRI findings in infants who had neonatal MMC surgery (depicted in the right two columns) as described earlier [27]. The middle right column in Fig. 1 illustrates MRI findings in patients who underwent neonatal MMC surgery and had posterior fossa decompression in relation to MRI findings in patients who had not had the decompression surgery (right column) as reported in the current study.

The cerebellar abnormalities reported in utero in fetuses with CII [27], have clinical consequences that appear when the patient is old enough to be examined and tested. The cerebellar atrophy leads to cognitive and motor deficits because there is no room for normal neural tissue expansion and physiological cerebellar growth. After birth, the cerebellar atrophy is not reversed by posterior fossa decompression.

If the cerebellar abnormalities and atrophy occur even after fetal surgical repair of the spinal lesion or posterior fossa decompression in infancy, how might they be ameliorated or minimized? The author proposes that early surgery to expand and enlarge the size of the posterior fossa (see the star symbol on the left in Fig. 1), to be performed initially as a pilot study in a select group of infants with CII who have a significantly small posterior fossa, 
may have the potential to prevent or decrease the severity of the clinical symptoms and signs related to CII, stop or reduce the cerebellar atrophy, and perhaps facilitate normal and unhindered growth of the hindbrain, which will likely lead to the preservation of its many functions. Furthermore, this surgery might have the added benefit of normalizing CSF flow within the posterior fossa in early infancy, thus allowing for more physiological hindbrain development to occur since the CSF contains growth factors that are important for brain development $[27,30]$.

The potential surgical benefits and risks should be weighed carefully before embarking on such an endeavor. More studies are needed first to address the optimum timing for such surgical procedure and in further defining the subset of CII patients who might potentially benefit. Investigating clinical and neuroradiological outcomes in relationship to the size of the posterior fossa and its contents in early infancy is a good starting point.

\section{Acknowledgments}

The author thanks Dr. M. Dennis from The Hospital for Sick Children, Toronto, Ontario, for her support and for her comments on earlier versions of the manuscript, and Dr. S.E. Blaser from The Hospital for Sick Children, Toronto, Ontario and Dr. M.E. Brandt from University of Texas Health Science Center, Houston, Texas for their help with the MRI data processing.

This work was supported in part by US National Institutes of Health Program Project [Grant P01 HD35946] "Spina bifida: Cognitive and neurobiological variability" to J. Fletcher and M. Dennis.

\section{References}

1. Wagner W, Schwarz M, Perneczky A. Primary myelomeningocele closure and consequences. Curr Opin Urol. 2002; 12:465-468. [PubMed: 12409873]

2. Barkovich, J. Pediatric neuroimaging. 4. Lippincott, Williams \& Wilkens; Philadelphia: 2005.

3. Harding, BN.; Copp, AJ. Malformations. In: Graham, DI.; Lantos, PL., editors. Greenfield's neuropathology. E. Arnold; London, UK: 2002. p. 376-386.

4. Juranek J, Salman MS. Anomalous development of brain structure and function in spina bifida myelomeningocele. Dev Disabil Res Rev. 2010; 16:23-30. [PubMed: 20419768]

5. Hori A. Chiari anomaly type II without cerebellar herniation. Acta Neuropathol. 2003; 105:193194. [PubMed: 12536232]

6. Brocklehurst G. A quantitative study of a spina bifida foetus. J Pathol. 1969; 99:205-211. [PubMed: 4917707]

7. Sener RN. Cerebellar agenesis versus vanishing cerebellum in Chiari II malformation. Comput Med Imaging Graph. 1995; 19:491-494. [PubMed: 8796972]

8. Boltshauser E, Schneider J, Kollias S, Waibel P, Weissert M. Vanishing cerebellum in myelomeningocoele. Eur J Paediatr Neurol. 2002; 6:109-113. [PubMed: 11995957]

9. Emery JL, Gadsdon DR. A quantitative study of the cell population of the cerebellum in children with myelomeningocele. Dev Med Child Neurol. 1975; 15(Suppl 29):20-25.

10. Variend S, Emery JL. The weight of the cerebellum in children with myelomeningocele. Dev Med Child Neurol. 1973; 15 (Suppl 29):77-83. [PubMed: 4354047]

11. Dennis M, Edelstein K, Hetherington R, Copeland K, Frederick J, Blaser SE, Kramer LA, Drake JM, Brandt M, Fletcher JM. Neurobiology of perceptual and motor timing in children with spina bifida in relation to cerebellar volume. Brain. 2004; 127:1-10.

12. Fletcher JM, Copeland K, Frederick JA, Blaser SE, Kramer LA, Northrup H, Hannay HJ, Brandt ME, Francis DJ, Villarreal G, Drake JM, Laurent JP, Townsend I, Inwood S, Boudousquie A, Dennis M. Spinal lesion level in spina bifida: a source of neural and cognitive heterogeneity. $\mathrm{J}$ Neurosurg (Pediatrics 3). 2005; 102:268-279.

13. Juranek J, Dennis M, Cirino PT, El-Messidi L, Fletcher JM. The cerebellum in children with spina bifida and Chiari II malformation: quantitative volumetrics by region. Cerebellum. 2010; 9 (2): 240-248. [PubMed: 20143197] 
14. Dennis M, Hopyan T, Juranek J, Cirino PT, Hasan KM, Fletcher J. Strong-meter and weak-meter rhythm identification in spina bifida meningomyelocele and volumetric parcellation of rhythmrelevant cerebellar regions. Ann NY Acad Sci. 2009; 1169:84-88. [PubMed: 19673758]

15. Gilbert JN, Jones KL, Rorke LB, Chernoff GF, James HE. Central nervous system anomalies associated with meningomyelocele, hydrocephalus, and the Arnold-Chiari malformation: reappraisal of theories regarding the pathogenesis of posterior neural tube closure defects. Neurosurgery. 1986; 18:559-564. [PubMed: 3714003]

16. Tsai T, Bookstein FL, Levey E, Kinsman SL. Chiari-II malformation: a biometric analysis. Eur J Pediatr Surg. 2002; 12(Suppl 1):S12-S18. [PubMed: 12541208]

17. Salman MS, Blaser SE, Sharpe JA, Dennis M. Cerebellar vermis morphology in children with spina bifida and Chiari type II malformation. Childs Nerv Syst. 2006; 22(4):385-393. [PubMed: 16374591]

18. Salman MS. The cerebellum in Chiari type II malformation. Neuroembryol Aging. 2008; 5:14-22.

19. Fletcher JM, McCauley SR, Brandt ME, Bohan TP, Kramer LA, Francis DJ, Thorstad K, Brookshire BL. Regional brain tissue composition in children with hydrocephalus: relationships with cognitive development. Arch Neurol. 1996; 53:549-557. [PubMed: 8660158]

20. Hasan KM, Eluvathingal T, Kramer LA, Ewing-Cobbs L, Dennis M, Fletcher JM. White matter microstructural abnormalities in children with spina bifida myelomeningocele and hydrocephalus: a diffusion tensor tractography study of the association pathways. J Magn Reson Imaging. 2008; 27:700-709. [PubMed: 18302204]

21. Stevenson KL. Chiari type II malformation: past, present, and future. Neurosurg Focus. 2004; 16(2):E5. [PubMed: 15209488]

22. Danzer E, Finkel RS, Rintoul NE, Bebbington MW, Schwartz ES, Zarnow DM, Adzick NS, Johnson MP. Reversal of hindbrain herniation after maternal-fetal surgery for myelomeningocele subsequently impacts on brain stem function. Neuropediatrics. 2008; 39(6):359-362. [PubMed: 19569004]

23. Huber-Okrainec J, Dennis M, Brettschneider J, Spiegler BJ. Neuromotor speech deficits in children and adults with spina bifida and hydrocephalus. Brain Lang. 2002; 80:592-602. [PubMed: 11896659]

24. Leigh, RJ.; Zee, DS. The neurology of eye movements. 4. Oxford University Press; New York: 2006.

25. Salman MS, Dennis M, Sharpe JA. The cerebellar dysplasia of Chiari II malformation as revealed by eye movements. Can J Neurol Sci. 2009; 36(6):713-724. [PubMed: 19960749]

26. Cai C, Oakes WJ. Herniation syndromes: the Chairi malformations (I and II). Semin Pediatr Neurol. 1997; 4(3):179-191. [PubMed: 9323788]

27. Danzer E, Johnson MP, Bebbington M, Simon EM, Wilson RD, Bilaniuk LT, Sutton LN, Adzick NS. Fetal head biometry assessed by fetal magnetic resonance imaging following in utero myelomeningocele repair. Fetal Diagn Ther. 2007; 22(1):1-6. [PubMed: 17003546]

28. Griffiths PD, Wilkinson ID, Variend S, Jones A, Paley MN, Whitby E. Differential growth rates of the cerebellum and posterior fossa assessed by post mortem magnetic resonance imaging of the fetus: implications for the pathogenesis of the chiari 2 deformity. Acta Radiol. 2004; 45(2):236242. [PubMed: 15191112]

29. Volpe JJ. Cerebellum of the premature infant: rapidly developing, vulnerable, clinically important. J Child Neurol. 2009; 24 (9):1085-1104. [PubMed: 19745085]

30. Mashayekhi F, Draper CE, Bannister CM, Pourghasem M, Owen-Lynch PJ, Miyan JA. Deficient cortical development in the hydrocephalic Texas (H-Tx) rat: a role for CSF. Brain. 2002; 125(Pt 8):1859-1874. [PubMed: 12135976] 


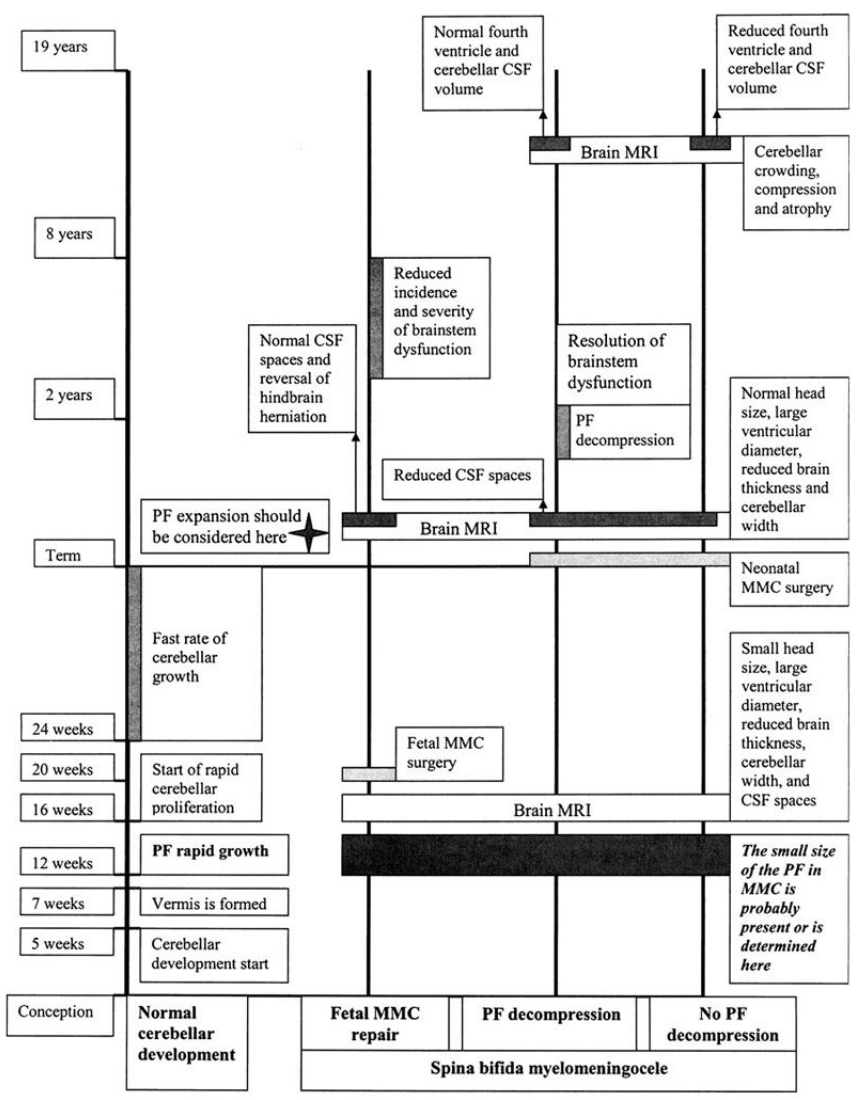

Fig. 1.

The diagram illustrates important milestones in posterior fossa $(P F)$ and cerebellar development (left column), and quantitative MRI findings in fetuses with myelomeningocele $(M M C)$ before and after either fetal MMC repair (left middle column) or postnatal MMC repair (right two columns). The right two columns also show the long-term quantitative MRI findings in CII patients with postnatal MMC repair who either had or did not have posterior fossa decompression for acute brainstem dysfunction. Both fetal MMC repair and posterior fossa decompression lead to healthier brainstem function. However, cerebellar atrophy is inevitable in all MMC patients, because the small size of the posterior fossa in MMC probably occurs very early in utero as shown in the figure (bold and italics box on the right). Early surgical expansion of the PF (box with the star symbol) is suggested to prevent or reduce the cerebellar atrophy in CII patients and facilitate a more physiological and unrestricted cerebellar growth. Information shown in this figure (not drawn to scale) is based on findings from this study and on information provided in references [17,18,22,27-29] 


\section{Table 1}

Demographic details of participants in the control group, CII group who did not have posterior fossa (PF) decompression, and CII group who had PF decompression

\begin{tabular}{llll}
\hline & Normal controls $[N=\mathbf{1 0}]$ & CII without PF decompression $[N=28]$ & CII with PF decompression $[\boldsymbol{N = 4 ]}$ \\
\hline Age in years & $12.7(2.2)$ & $13.2(2.9)$ & $13.1(3.3)$ \\
Gender (M/F) & $5 / 5$ & $14 / 14$ & $4 / 0$ \\
Spinal lesion level (U/L) & Not applicable & $7 / 21$ & $2 / 2$ \\
Cerebellar vol. & $154161(13804)$ & $113704(29248)$ & $123880(25786)$ \\
Medial cerebellar vol. & $20070(3081)$ & $18484(5058)$ & $22254(3663)$ \\
Lateral cerebellar vol. & $134091(11201)$ & $95220(24608)$ & $101626(22442)$ \\
Total cerebellar CSF & $6305(1738)$ & $4636(1602)$ & $6391(993)$ \\
Cerebellar gray matter vol. & $111080(10445)$ & $81426(22584)$ & $86715(18670)$ \\
Cerebellar white matter vol. & $36776(4372)$ & $27642(7210)$ & $30774(7891)$ \\
\hline
\end{tabular}

Mean cerebellar volumes (cubic millimeters, $\mathrm{mm}^{3}$ ) and its components are displayed (SD)

$U$ upper spinal lesion level (T12 and above), $L$ lower spinal lesion level (L1 and below), vol. volume 